\title{
Competencias de enfermería en el tratamiento no farmacológico del delirium en el adulto mayor
}

\section{Nursing competences in the non-pharmacological treatment of delirium in the elderly.}

\author{
Olivia Milagros Pérez-Solís ${ }^{1}$ \\ Sylvia Claudine Ramírez-Sánchez ${ }^{2}$ \\ Olga Lozano-Rangel 3
}

1 Instituto Mexicano del Seguro Social, Hospital de Cardiología, Centro Médico Nacional Siglo XXI, Clínica de geriatría

2 Instituto Mexicano del Seguro Social, Coordinación de Investigación en Salud, Unidad de Investigación Epidemiológica y Servicios de Salud Ciudad de México, México.

3 Instituto Mexicano del Seguro Social, Hospital de Cardiología Centro Médico Nacional Siglo XXI, Dirección de Enfermería.

Recibido el: 16 de febrero de 2019

Aceptado el: 22 de febrero de 2019 


\section{RESUMEN}

Introducción: El personal de enfermería es un elemento primordial en la atención del adulto mayor con Delirium, destacando no solo el conocimiento sino la habilidad y la actitud para el abordaje del tratamiento no farmacológico. Las competencias permiten identificar áreas de oportunidad que direccionen estrategias para elevar la calidad de atención hacia los derechohabientes.

Objetivo: Establecer el nivel de competencias del personal de enfermería en el tratamiento no farmacológico del delirium, en el adulto mayor en una unidad de tercer nivel.

Material y métodos: estudio transversal analítico, se llevó acabo de octubre a noviembre del 2018, en áreas de hospitalización, urgencias, terapia intensiva cardiovascular, terapia intensiva post quirúrgica, y rol suplente del Hospital de Cardiología del Centro Médico Nacional Siglo XXI. Se diseñó un instrumento con 48 ítems, dividido en dos secciones: la primera datos sociodemográficos, datos laborales y capacitación (21 ítems); la segunda con caso clínico estructurado (27 ítems) que evalúa las fases de conocimiento, habilidades y actitudes, valorando las competencias de acuerdo al modelo de Patricia Benner en sus cinco niveles: novato $(<6)$, principiante avanzado (7 -12), competente (13-18), eficiente19-25) y experto (26-27). Se validó el instrumento en ronda de expertos 3 de 5, Alpha de Cronbach .814. Se utilizó estadística descriptiva y no paramétrica, prueba de Kruskall-Wallis, r Sperman y chi cuadrada.

Resultados: se entrevistaron 110 enfermeras. La edad, la media fue de $40.71 \pm 9.13$, el sexo femenino conformó el 70\%, el nivel académico licenciatura reflejo el 35.5\%. Los niveles de competencia fueron: principiante el $1 \%$, competente $39 \%$ y eficiente $60 \%$, según las fases que integran las competencias las calificaciones: conocimiento 7.1, habilidades 6.9, y actitudes 7.9. Se correlacionó el nivel de competencia con el grado académico $(\mathrm{p}<0.005)$.

Conclusiones: El personal de enfermería, su nivel de competencia fue eficiente. Se identificó un área de oportunidad respecto a las habilidades que se deben poseer en el 
tratamiento no farmacológico del Delirium, lo que nos permitirá que los procesos de enseñanza aprendizaje sea redirigidos en el personal de enfermería.

Palabras clave: Enfermería, Competencias, Delirium en el adulto mayor.

\section{SUMMARY}

Introduction: The nursing staff is a primary element in the care of the elderly with Delirium, highlighting not only knowledge but also the ability and attitude to approach non-pharmacological treatment. The competences allow to identify areas of opportunity that direct strategies to raise the quality of attention to the beneficiaries.

Objective: To establish the competence level of the nursing staff in the nonpharmacological treatment of delirium, in the elderly in a third level unit.

Material and methods: an analytical cross-sectional study was carried out from October to November 2018, in areas of hospitalization, emergency, intensive cardiovascular therapy, intensive post-surgical therapy, and alternate role of the Cardiology Hospital of the National Medical Center Siglo XXI. An instrument with 48 items was designed, divided into two sections: the first sociodemographic data, labor data and training (21 items); the second with a structured clinical case (27 items) that evaluates the phases of knowledge, skills and attitudes, valuing the competences according to the model of Patricia Benner in its five levels: novice $(<6)$, advanced beginner (7 -12), competent (13-18), efficient19-25) and expert (26-27). The instrument was validated in expert round 3 of 5, Alpha de Cronbach .814. We used descriptive and non-parametric statistics, Kruskall-Wallis test, Sperman test and square chi test.

Results: 110 nurses were interviewed. The age, the average was of $40.71 \pm 9.13$, the feminine sex formed $70 \%$, the academic level of the degree reflected $35.5 \%$. The levels of competence were: beginner $1 \%$, competent $39 \%$ and efficient $60 \%$, according to the phases that integrate the competences the qualifications: knowledge 7.1 skills 6.9 , and attitudes 7.9. The level of competence was correlated with the academic degree ( $p$ $<0.005)$. 
Conclusions: The nursing staff, their level of competence was efficient, in the nonpharmacological treatment of Delirium. An area of opportunity was identified with respect to the skills that must be possessed in the non-pharmacological treatment of Delirium, which will allow the teaching-learning processes to be redirected in the nursing staff.

Keywords: Nursing, Competencies, Delirium in the elderly.

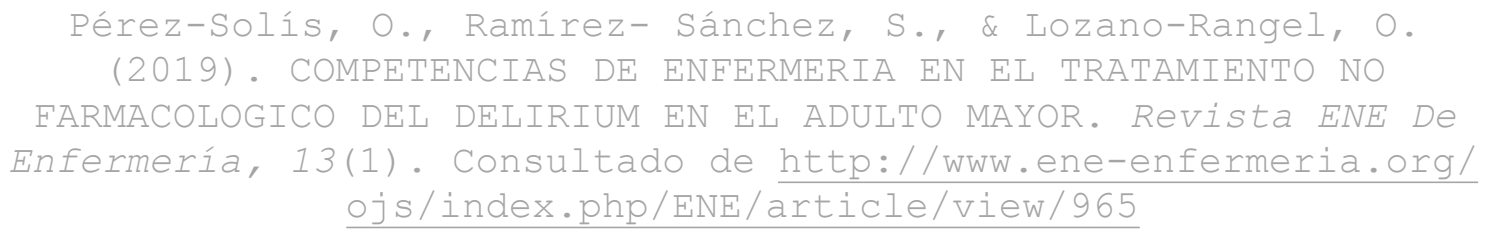




\section{INTRODUCCIÓN}

Conforme a las proyecciones que estima el Consejo Nacional de Población (CONAPO), en 2017 residen en el país de México 12, 973411 personas de 60 años y más, de los cuales $53.9 \%$ son mujeres y $46.1 \%$ son hombres, de acuerdo con la Organización de las Naciones Unidas (ONU), su tendencia esperada en los próximos años la colocan como una de las transformaciones sociales de mayor trascendencia del siglo XXI.(1) Los derechohabientes de los servicios de salud en la mayoría son adultos mayores, a nivel mundial se registran con el 55\%, y en México es de $60 \%$ acompañados con patologías múltiples(1).

Una de las complicaciones con mayor morbimortalidad en la población geriátrica hospitalizada es el Delirium lo que origina largas estancias hospitalarias en el $20 \%$ de los 12.5 millones de adultos mayores de 65 años que se hospitalizan en Estados Unidos, elevando los costos de atención en 2,500 dólares por paciente, sin tomar en cuenta que algunos requerirán a su egreso ser nuevamente re hospitalizados, rehabilitados, institucionalizados y auxiliados por cuidadores formales o informales de salud.

A nivel hospitalario la incidencia es variable y depende de la población estudiada, siendo del 18 a 35\%. En México, las instituciones de salud pública han reportado una prevalencia de delirium del $38.3 \%$ de los adultos mayores hospitalizados y una incidencia del $12 \%$, de los cuales casi la mitad tienen como antecedentes demencia, dolor no controlado o un procedimiento quirúrgico reciente.

Cabe resaltar que de aquellos pacientes hospitalizados con delirium, la mitad ingreso con delirium y la otra mitad la desarrolló durante la hospitalización. Pese a su alta frecuencia, éste no es diagnosticado por los médicos en el 50 al $80 \%$ de los casos y en enfermería más del $50 \%$ no es reconocido como diagnóstico así mismo no es intervenido eficazmente. Además, es importante enfatizar que el delirium durante la hospitalización es prevenible casi en el $40 \%$ de los casos. El delirium se asocia a una alta mortalidad, siendo del $34 \%$ en pacientes hospitalizados (1). La Unidad Médica de 
Alta Especialidad (UMAE) Hospital de Cardiología C.M.N. Siglo XXI, es una unidad de tercer nivel que atiende a una población de adultos mayores en más del $80 \%$ con enfermedades cardiovasculares que se ven potencializadas por las complicaciones del diagnóstico de base. El Delirium, en el Registro de productividad del programa de atención geriátrica 2016- 2017 de enfermería, reportó que durante ese año 1,800 adultos mayores fueron atendidos en hospitalización de ellos 1,680 fueron pacientes geriátricos registrando que el $40 \%$ presentó delirium.

Ante esa situación, nos encontramos ante la necesidad de una atención especializada para mejorar la atención a este grupo etario, es por ello que surge dentro del Instituto Mexicano del Seguro Social (IMSS) el programa de GERIATRIMSS el 25 de Noviembre del 2010, integrado por 4 estrategias fundamentales: a)fortalecimiento del programa PREVENIMSS para el envejecimiento saludable del adulto mayor, b)implementación y fortalecimiento de la atención geriátrica en las unidades médicas del Instituto, $c$ ) formación y capacitación en geriatría y d) desarrollo de la investigación clínica en envejecimiento a través de la formación y capacitación del personal especializado, como una área de oportunidad que requiere de esfuerzo para mejorar y ampliar su campo de acción.

Como integrante del equipo multidisciplinario, el personal de enfermería es un elemento clave en la atención oportuna del adulto mayor con Delirium siendo el encargado de realizar las intervenciones de prevención, diagnóstico enfermero y tratamiento no farmacológico (estrategias clínicas, conductuales y ambientales) por lo que el personal de enfermería debe poseer un nivel de competencia que contribuyan a una atención de calidad.

El Consejo Internacional de Enfermería (CIE) define competencia como: "Un nivel de realizaciones que demuestra la aplicación efectiva de los conocimientos, capacidades y juicio" que el personal de enfermería realiza a nivel laboral, refleja comprensión, conocimiento y juicio además de una importante gama de capacidades cognitivas, psicomotoras e interpersonales, éstas competencias son las que en el ejercicio de la práctica o su lugar laboral vive en instituciones de salud, en la comunidad y en todo el mundo para poder suministrar unos cuidados de calidad (1) 
Al respecto Patricia Benner apuesta por el conocimiento dirigido a la práctica. $(1,2)$ Benner sostiene que el conocimiento práctico puede ampliar la teoría o desarrollarse antes que las fórmulas científicas, dado que las situaciones clínicas siempre son variadas y complicadas de lo que la teoría muestra. Benner adopta el modelo de Dreyfus y Dreyfus de adquisidor y desarrollo de habilidades a la práctica de la enfermera clínica, Stuart y Hubert Dreyfus, ambos profesores de la Universidad de California en Berkeley, desarrollaron este modelo situacional que describe los cinco niveles de adquisidor y desarrollo de habilidades novato(principiante) principiante avanzado, competente, eficiente y experto. (Figura 1). ${ }^{1}$

\begin{tabular}{|c|c|c|c|c|}
\hline \multicolumn{5}{|c|}{ Etapas } \\
\hline Novato & $\begin{array}{c}\text { Principiante } \\
\text { avanzado }\end{array}$ & Competente & Eficiente & Experto \\
\hline $\begin{array}{l}\text { Aprende las } \\
\text { reglas básicas. } \\
\text { Quiere lograr } \\
\text { una tarea } \\
\text { específica a la } \\
\text { vez. } \\
\text { Sigue las } \\
\text { instrucciones } \\
\text { paso a paso. }\end{array}$ & $\begin{array}{l}\text { Necesidad de } \\
\text { obtener información } \\
\text { al instante. } \\
\text { No globaliza. } \\
\text { Dificultad para lidiar } \\
\text { con problemas. } \\
\text { Comienza a aplicar } \\
\text { reglas de acuerdo a } \\
\text { la situación. } \\
\text { No asimila su grado } \\
\text { de responsabilidad. }\end{array}$ & $\begin{array}{l}\text { Plantea metas a } \\
\text { largo plazo. } \\
\text { Resuelve } \\
\text { problemas. } \\
\text { Busca consejos de } \\
\text { expertos. } \\
\text { Capacidad para } \\
\text { comprender } \\
\text { determinadas } \\
\text { circunstancias. } \\
\text { Aplica modelos } \\
\text { aprendidos. }\end{array}$ & $\begin{array}{l}\text { Quiere } \\
\text { comprender su } \\
\text { área de trabajo. } \\
\text { Aprende de las } \\
\text { experiencias de } \\
\text { otros. } \\
\text { Entender y aplicar } \\
\text { al máximo. } \\
\text { No le agradan las } \\
\text { soluciones fáciles. }\end{array}$ & $\begin{array}{l}\text { Gran desarrollo } \\
\text { de la intuición. } \\
\text { Es una fuente } \\
\text { de } \\
\text { conocimiento. } \\
\text { Busca mejores } \\
\text { métodos. } \\
\text { Analiza más allá } \\
\text { de las reglas } \\
\text { establecidas. }\end{array}$ \\
\hline
\end{tabular}

Figura l. Origen y Clasificación de la Teoría de Patricia Benner

En el área de enfermería se requiere de una capacidad para el desarrollo de competencias construyéndose a lo largo de una vida laboral a partir de experiencias y practica con personas que integran el equipo de trabajo, los pacientes y su familia, siendo el cuidado la razón de la ciencia que nos permite adquirir nuevas habilidades. 
La evaluación por medio de la simulación clínica requiere definir los escenarios específicos integrales que correspondan a las competencias para mejorar el proceso en la toma de decisiones, la comunicación entre el equipo, la optimización de recursos y la posibilidad de error.

La importancia que esto conllevó a la elaboración de esta investigación se debe a que se desconoce el nivel de competencias del profesional de enfermería y siendo ésta la manera de identificarlo. Los resultados nos permitieron describir las características sociodemográficas, así como datos para establecer el nivel en que se encontró el profesional de enfermería en una unidad de tercer nivel.

La formación basada en competencias responde a las demandas de atención en salud, permitiendo obtener información para identificar necesidades de aprendizaje, técnicas, y procedimientos para la mejora de calidad en la atención en los servicios de salud. 


\section{MATERIAL Y MÉTODOS}

Se realizó un estudio transversal analítico, de octubre a noviembre del 2018, en la Unidad Médica de Alta Especialidad Hospital de Cardiología del Centro Médico Nacional Siglo XXI (UMAE HC CMN SXXI).

La muestra se conformó por $n=110$ enfermeras(o) del total del personal de enfermería, adscritos a los servicios de hospitalización, urgencias, terapia intensiva cardiovascular, terapia intensiva post quirúrgica y rol suplente, de diferente categoría jerárquica: auxiliar de enfermera general, enfermera general, enfermera especialista intensivista y jefes de piso de los tres turnos.

Para el cálculo del tamaño de la muestra se utilizó la fórmula de estimación de proporciones, la selección se hizo por muestreo aleatorio estratificado por categoría contractual.

Se diseñó un instrumento con el título de "Competencias del personal de enfermería en el tratamiento no farmacológico del Delirium en el adulto mayor en un hospital de tercer nivel", con 48 ítems, dividido en dos secciones: la primera con datos sociodemográficos, datos laborales y capacitación (21 ítems); la segunda con caso clínico estructurado (27 ítems) que evalúa las fases de conocimiento, habilidades y actitudes, valorando las competencias de acuerdo al modelos de Patricia Benner en sus cinco niveles: novato $(<6)$, principiante avanzado $(7-12)$, competente $(13-18)$, eficiente19-25) y experto (26-27).

La validación del instrumento fue por ronda de expertos 3 de 5, Alpha de Cronbach .814. Antes de la recolección de datos, se informó sobre el objetivo del estudio a las subjefes de enfermería, con la finalidad de concientizar acerca la medición de las competencias en el personal adscrito a los servicios.

La invitación al personal de enfermería fue verbal, con la correcta explicación sobre el objetivo del estudio, posteriormente; se les entregó carta de consentimiento informado, se les hizo hincapié en que la información que proporcionaran seria confidencial y para fines estadísticos. 
El instrumento fue auto aplicado con un tiempo estimado para responderlo de 15 a 20 min. Por lo tanto, se respetó el derecho del personal con base en los aspectos éticos considerados en la Ley General de Salud en materia de investigación en salud, ${ }^{1}$ así como en la Declaración de Helsinki ${ }^{2}$ por lo cual el personal participante tuvo la libertad de retirarse en el momento en que lo deseara.

Se determinó esta investigación como de mínimo riesgo, pues consistió en contestar un instrumento. El proyecto de investigación fue evaluado por el Sistema de Registro Electrónico de la coordinación de investigación con número de folio F-2018-3604-32 y autorizado por el Comité Local de Investigación con número R-2018-3604-024.

Se elaboró una base de datos en el programa SPSS, versión 23, donde se capturó la información de los instrumentos. Asimismo, se utilizó estadística descriptiva y no paramétrica, prueba de Kruskall-Wallis, r Sperman y chi cuadrada. 


\section{RESULTADOS}

Se entrevistó a 110 enfermeras de las áreas de urgencias, hospitalización, terapia intensiva cardiovascular, terapia intensiva post quirúrgica y rol suplente, del Hospital de Cardiología del Centro Médico Nacional Siglo XXI.

El promedio de edad del personal de enfermería fue de $40.71 \pm 9.13$, este en su mayoría del sexo femenino $70 \%$ y casada(o) 50\%. (Figura 2)

\begin{tabular}{|c|c|c}
\hline Género & Frecuencia & Porcentaje (\%) \\
\hline Femenino & 77 & 70 \\
\hline Masculino & 33 & 30 \\
\hline Estado civil & & 33.6 \\
\hline Soltero & 37 & 50.0 \\
\hline Casado & 55 & 9.1 \\
\hline Unión libre & 10 & 5.5 \\
\hline Divorciado & 6 & 1.8 \\
\hline Viudo & 2 & 28.2 \\
\hline Grado académico & 31 & 27.3 \\
\hline Técnica & 30 & 35.5 \\
\hline Curso pos técnico & 39 & 7.3 \\
\hline Licenciatura & 8 & 1.8 \\
\hline Posgrado & 2 & \\
\hline Maestría & & \\
\hline
\end{tabular}

Figura 2. Datos sociodemográficos del personal de enfermería $(n=110)$ Fuente: Instrumento" Competencias del profesional de enfermería en el tratamiento no farmacológico del delirium en el Adulto mayor en una unidad de tercer nivel

El turno que prevalece en el estudio es el vespertino $41.8 \%$, en categoría la enfermera especialista intensivista en $40.9 \%$ en comparación con el porcentaje de las jefes de piso $11.8 \%$, y el servicio de mayor participación en los cuestionarios es terapias intensivas con el 40.9\% y hospitalización 36.4\%. (igura 3 ) 


\begin{tabular}{|c|c|c|}
\hline Variable & Frecuencia & $\%$ \\
\hline \multicolumn{3}{|l|}{ Turno } \\
\hline Matutino & 41 & 37.3 \\
\hline Vespertino & 46 & 41.8 \\
\hline Nocturno & 23 & 20.9 \\
\hline \multicolumn{3}{|l|}{ Categoría } \\
\hline Auxiliar de enfermera general & 21 & 19.1 \\
\hline Enfermera general & 31 & 28.2 \\
\hline Enfermera especialista intensivista & 45 & 40.9 \\
\hline Jefe de piso & 13 & 11.8 \\
\hline \multicolumn{3}{|l|}{ Servicio } \\
\hline Hospitalización & 40 & 36.4 \\
\hline Urgencias & 12 & 10.9 \\
\hline Terapia intensiva & 18 & 16.4 \\
\hline Post quirúrgica & 27 & 24.5 \\
\hline Rol suplente & 13 & 11.8 \\
\hline
\end{tabular}

Figura 3. Aspectos laborales del personal de enfermería adscritos a los servicios de Hospitalización, urgencias, terapia intensiva, post quirúrgica, rol suplente. Fuente: Instrumento" Competencias del profesional de enfermería en el tratamiento no farmacológico del delirium en el Adulto mayor en una unidad de tercer nivel

La antigüedad laboral reflejo una mediana $(\mathrm{Me})$ de 15 , con valor mínimo de 1 y máximo de 37 años, la antigüedad institucional una mediana $\left(\mathrm{M}_{\mathrm{e}}\right)$ de 11 , con valor mínimo de 1 y máximo de 37 años, la antigüedad del servicio mediana (Me) de 1, valor mínimo menor a 1 mes y máximo de 25 años.

La capacitación continua permite mejorar el desempeño del personal en las tareas clínicas, pero al respecto solo el $4.5 \%$ reportó que ha recibido algún tipo de capacitación en el área de geriatría, en comparación con el 95.5\% que no ha recibido.

En general el personal de enfermería obtuvo un nivel de competencia eficiente en un $60 \%$, de este personal el $62 \%$ se desempeña en el servicio de hospitalización, y el $55.5 \%$ en la terapia post quirúrgica. (Figura 4). 


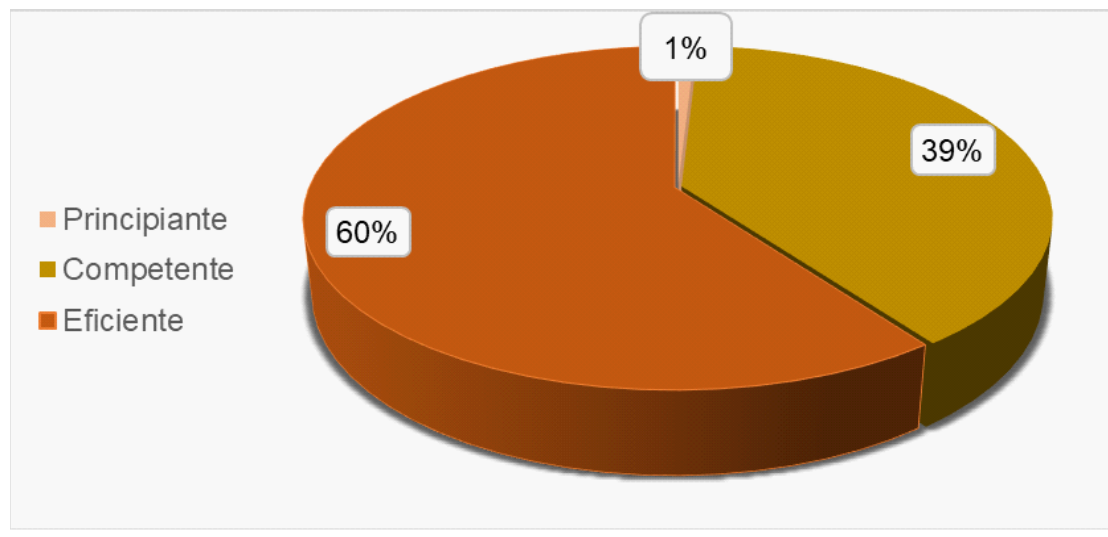

Figura. 4 Nivel de competencias del personal de enfermería en el tratamiento no farmacológico del Delirium en el adulto mayor(n=110)Fuente: Instrumento" Competencias del profesional de enfermería en el tratamiento no farmacológico del delirium en el Adulto mayor en una unidad de tercer nivel

En las fases de las competencias del personal de enfermería la calificación en general fue en conocimiento 7.1, habilidades 6.9, y actitud 7.9. (Figura. 5).

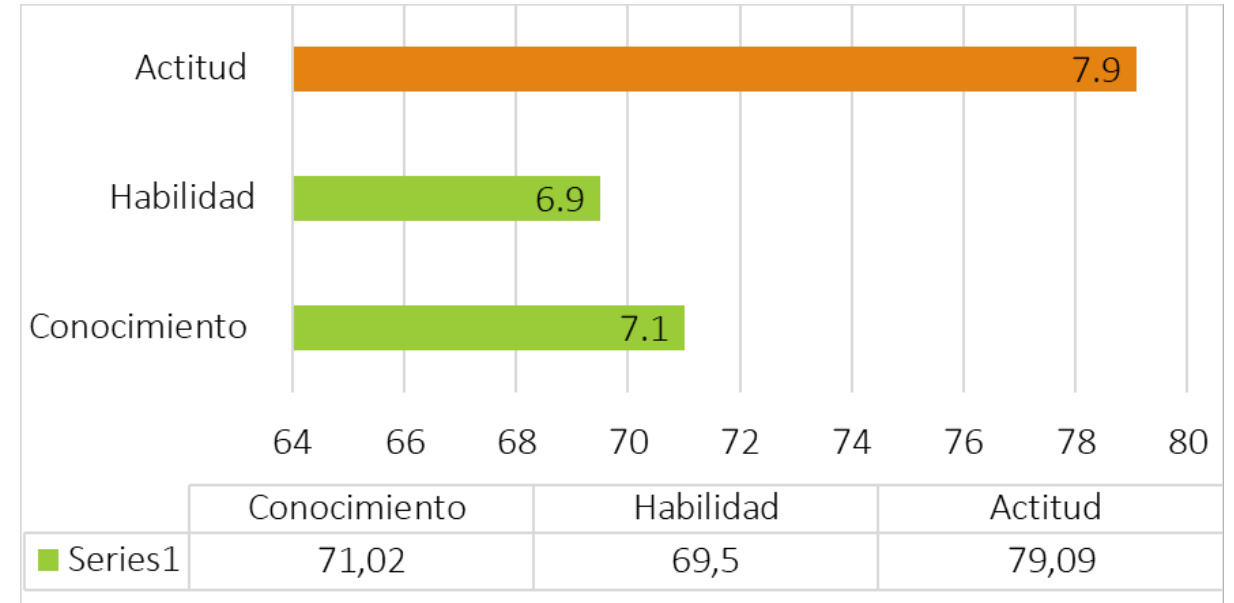

Figura. 5. Calificación de las fases de competencias del personal de enfermería en el tratamiento no farmacológico del Delirium en el adulto mayor( $\mathrm{n}=110)$ Fuente: Instrumento" Competencias del profesional de enfermería en el tratamiento no farmacológico del delirium en el Adulto mayor en una unidad de tercer nivel 
En relación con el grado académico y el nivel de competencia, fue más eficiente el personal con formación profesional de licenciatura $(23.6 \%)$ seguido de formación técnica $(15.40 \%)$ en el mismo nivel de competencia, pero en mínima proporción estuvo el personal con maestría (0.9\%). (Fig. 6.)

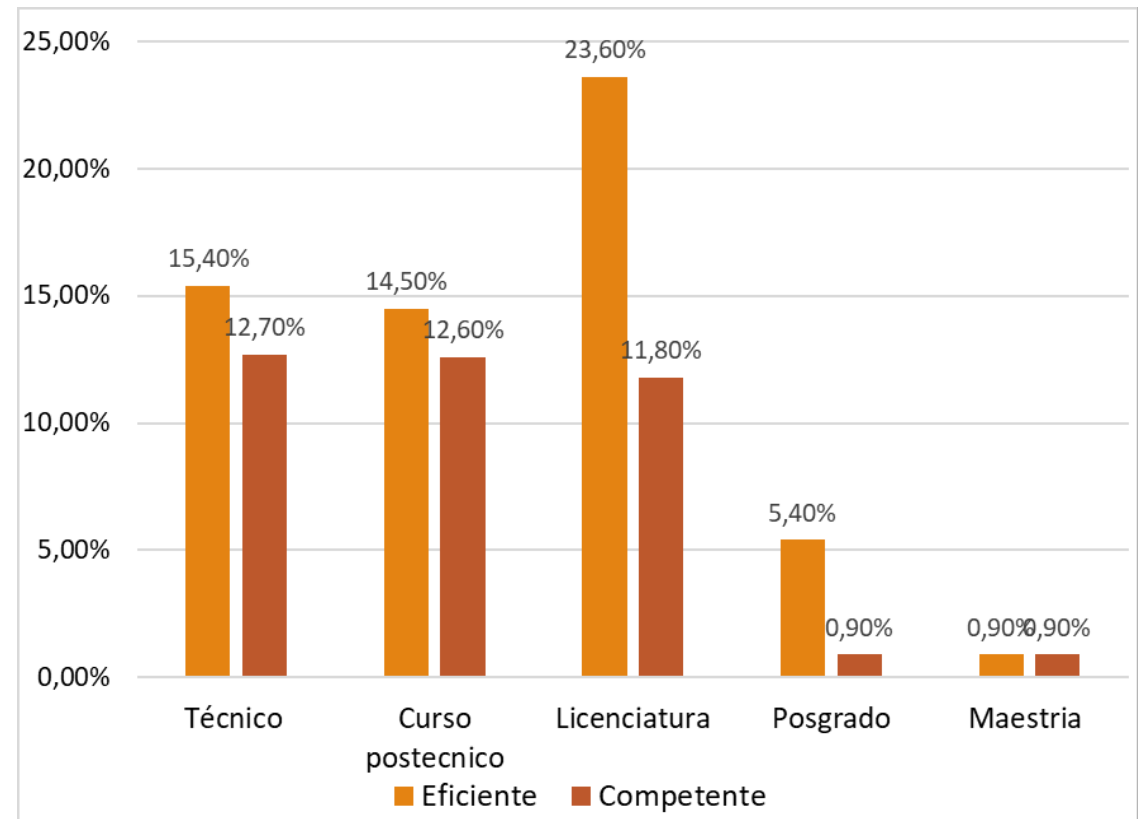

Figura. 6. Nivel de competencia clínica en relación al nivel académico del personal de enfermería en el tratamiento no farmacológico del Delirium. Fuente: Instrumento" Competencias del profesional de enfermería en el tratamiento no farmacológico del delirium en el Adulto mayor en una unidad de tercer nivel. Asociación Nivel de Competencia y Nivel Académico. Prueba estadística X2 (Chi cuadrada) p.000

Sin duda el grado académico es un factor predominante para un buen desempeño y desarrollo de las competencias clínicas ya que determino la asociación siendo esta (p. 000) utilizando la prueba estadística de Chi cuadrada.

\section{Estadísticos de prueba}

\begin{tabular}{|l|r|r|}
\hline & \multicolumn{1}{|c|}{$\begin{array}{c}\text { nivel } \\
\text { académico }\end{array}$} & competencia \\
\hline Chi-cuadrado & $46.818^{\mathrm{a}}$ & $59.255^{\mathrm{b}}$ \\
$\mathrm{gl}$ & 4 & 2 \\
Sig. asintótica & .000 & .000 \\
\hline
\end{tabular}


En cuanto al nivel de competencia en relación con la antigüedad laboral, se observó que a menor antigüedad mayor nivel de competencia, siendo esta estadísticamente no significativa a mayor antigüedad laboral mayor nivel de competencia (Fig.7).

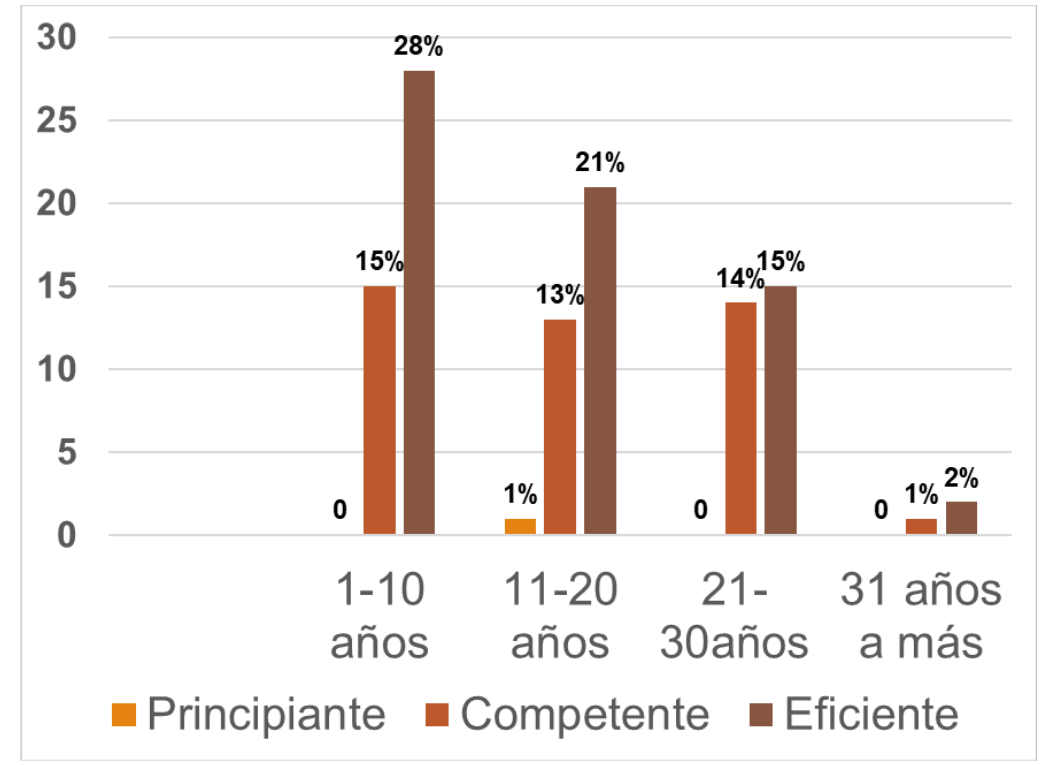

Figura 7. Nivel de competencia clínica en relación con la antigüedad laboral del personal de enfermería en el tratamiento no farmacológico del Delirium.Fuente: Instrumento" Competencias del profesional de enfermería en el tratamiento no farmacológico del delirium en el Adulto mayor en una unidad de tercer nivel. Asociación Nivel de competencia y antigüedad laboral. Prueba estadística H. Kruskal Wallis p.374 


\section{DISCUSIÓN}

Los procesos de enseñanza-aprendizaje en el área de salud tienen como objetivo primordial el desarrollo de habilidades procedimentales y la adquisición de diversas competencias a través de la experiencia clínica, siendo la práctica reflexiva un elemento en este proceso. ${ }^{2}$

En nuestro estudio el sexo femenino fue del $70 \%$ mientras que en las investigaciones realizadas de Duque et al ${ }^{1}$ y Ramos Melgar et al ${ }^{3}$ fue del 61\%, y 77\%, siendo que el personal de enfermería sigue siendo representada por mujeres.

En relación con los niveles de competencias los más representativos fueron eficiente $60 \%$, competente $39 \%$, principiante $1 \%$, existiendo diferencia con el nivel de eficiente reportado en un $75 \%$ para prevenir ulceras por presión, y con el $74 \%$ en la atención del paciente en Código Infarto puerta-balón-primaria. La relación del nivel de competencia clínica en un nivel eficiente con el grado académico fue favorable en el caso del personal licenciatura 35.4\%, coincidiendo con Ramos Melgar, en relación con las fases de las competencias la calificación en general fue en conocimiento 7.1, habilidades 6.9, y actitud 7.9, existiendo similitudes con Duque que tanto las habilidades y el conocimiento son de rango bajo, habilidad 4.7, 6.2 conocimiento y existiendo diferencia en la actitud de 5.0.

La enfermera en el tratamiento no farmacológico del Delirium en el adulto mayor debe tener la capacidad de liderar los procesos, brindar respuestas inmediatas, eficaces y asertivas las cuales cumplan con la atención integral durante el desarrollo y la evaluación del servicio prestado, asumiendo todas las competencias en sus tres dimensiones lo cual se relaciona con Patricia Benner, donde se destaca que el personal de enfermería enfrente nuevos retos y se destaque en un área determinada, con responsabilidad, autonomía, rapidez y veracidad identificándola como experta. (1) Es motivo de reflexión, si las calificaciones obtenidas en actitud, conocimiento y habilidad son suficientes para que el personal de enfermería proporcione el cuidado y atención que requieren los pacientes con esta patología. 
Sin embargo, se encuentra que el hecho de que el personal tenga una actitud favorable es una fortaleza ya que sería posible capacitarla en éste área, convirtiéndose así en una oportunidad que daría pie a una nueva pregunta de investigación.

\section{CONCLUSIÓN}

El nivel de competencia del personal de enfermería fue eficiente, aceptando la hipótesis alterna y observando que predominó la actitud, a diferencia de la habilidad y el conocimiento. Este hecho es importante para continuar evaluando las fases de las competencias en cuanto a la habilidad y el conocimiento, estableciendo estrategias de aprendizaje practico que facilite al personal de enfermería las herramientas para beneficio en la atención del paciente con Delirium. Así como diseñar un programa educativo que permita incrementar el área de oportunidad identificada en la fase de habilidad.

En cuanto a la antigüedad laboral se identifica que el personal de enfermería debe ser incentivado para actualizarse de manera continua, dado que durante los primeros años la capacitación se considera activa y a medida que pasa el tiempo el interés comienza a declinar, teniendo así experiencia mas no siempre conocimientos.

\section{AGRADECIMIENTOS}

Al Lic. Héctor Genis Zárate médico geriatra, Lic. José Enrique Cruz Aranda médico geriatra, Lic. Alejandro Jahén García Delgado médico geriatra, Mtra. Ana Belem López Morales Coordinadora Nivel Nacional del Programa de GERIATRIMSS en enfermería, Lic. Juan Carlos López Moctezuma enfermero geriatra, Lic. Enf. María de los Ángeles Ayala Cervantes enfermera Jefe de piso, Dr. Omar Pérez Enríquez, a las enfermeras(o) de los servicios de hospitalización, terapia intensiva coronaria, terapia intensiva post quirúrgica, $\mathrm{y}$ urgencias. 


\section{BIBLIOGRAFÍA}

1. Organización de las Naciones Unidas ONU ; (s/f). Envejecimiento. Recuperado el 6 de junio de 2017, de: http://www.un.

2. org/es/sections/issues-depth/ageing/index.html.

2. Ikeda $\mathrm{N}$ et al. What has made the population of japan healthy? Lancet $2011 ; 378$ : 1.094-105.

3. Witlox J, Eurelings L, De Jonghe J, Kalisvaart K, Eikelenboom P, Van Gool W. Delirium in Elderly Patients and the Risk of Post discharge Mortality, Institutionalization, and Dementia A Meta-analysis. . JAMA. 2010;304(4);443-451

4. Consejo Internacional de Enfermeras, 2000(a) Competencias internacionales para la enfermera generalista: Especificación para un proyecto. Documento inédito, CIE, Ginebra.

5. Benner P. Práctica de enfermería progresiva. Barcelona: Grijalbo, 1987.

6. Schón DA. El profesional reflexivo. Madrid: Paidós, 1992.

7. Marriner. A. Raile, M .Modelos y Teorías en Enfermería. Sexta edición, España. Editorial Harcourt Brace 2007:140-166.

8. Secretaria de Salud (SS). Ley de Salud en Materia en Investigación para la Salud. México: SS pp 1-23. Disponible en htpp: //www.salud. gob.mx/unidades/cdi/nom/ compi/rlgsmis.html.; 28 de Julio 2018.

9. Asociación Médica Mundial (AMM). Declaración e Helsinki de la AMM. Principios éticos para las investigaciones médicas en seres humanos. Brasil: AMM; 2013. Disponibles en htpp: //Www. iscii.es/ISCIII/es/contenidos/fd- investigación/fd evaluación/fd evaluación-ética-investigación/Declaración de Helsinki-2013-Esp.pdf; 23 de Julio 2018; 
10. Latrach- Ammar C, Febre N, Demandes I, Araneda J, González I. Importancia de las competencias en la formación de enfermería. Aquichan, Chia Colombia. 2011: 305-315.;consulado 27 de Noviembre 2018; Disponible en htpp://redalyc.org/pdf/ 741/74121424006.pdf.

11. Duque-Ruelas P, Romero Quechol G, Martínez-Olivares M. Competencias de enfermería para prevenir ulceras por presión. Rev Enferm Inst Mex Seguro Soc; 2015;23(3):169-9.

12. Ramos-Melgar M, Martínez Olivares M, Romero Quechol G, Salgado-Marín M. Competencia clínica del profesional de enfermería en la atención del paciente en Código Infarto puerta-balón-primaria. Rev Enferm Inst Mex Seguro Soc; 2017; 25(4): 279-84.

13. Carrillo-Algarra AJ, García Serrano L, Cárdenas Orjuela CM, Díaz Sánchez IR, Yabrudy-Wilches N. LA FILOSOFIA DE Patricia Benner y la práctica clínica. Enferm Glob.2013;12(32):346-61;consultado 28 de Noviembre 2018.Disponible en htpp:// www.redalyc.org/articulo.oa?id=365834851020. 